\title{
Relación entre: orientación de meta y afinidad del usuario por la didáctica y características del docente en clase de aeróbicos
}

\section{Relationship between: goal orientation and affinity of the user for the didactics and characteristics of the teacher in an aerobics class}

\author{
Jhonnatan Andrés Díaz (iD; Luz Amelia Hoyos-Cuartas (iD
}

'Universidad Pedagógica y Tecnológica de Colombia. Bogotá, Colombia. Jhonnatan.díaz@uptc.edu.co; Ihoyos@pedagogica.edu.co

Cómo citar: Díaz, J.A.; Hoyos-Cuartas, L.A. 2021. Relación entre: orientación de meta y afinidad del usuario por la didáctica y características del docente en clase de aeróbicos. Rev. Digit. Act. Fis. Deport. 7(2):e1932. http:// doi.org/10.31910/rdafd.v7.n2.2021.1932

Artículo de acceso abierto publicado por Revista Digital: Actividad Física y Deporte, bajo una licencia Creative Commons CC BY-NC 4.0

Publicación oficial de la Universidad de Ciencias Aplicadas y Ambientales U.D.C.A, Institución de Educación Superior Acreditada de Alta Calidad por el Ministerio de Educación Nacional

Recibido: marzo 1 de 2021 Aceptado: marzo 29 de 2021 Editado por: Álvaro José Gracia Díaz

\section{RESUMEN}

Introducción: Los aeróbicos son una práctica de ejercicio físico de forma grupal y musicalizada, cuyo objetivo es trabajar el sistema cardiovascular, la memoria motriz y la coordinación. En Bogotá, esta práctica es muy popular en lugares como gimnasios, institutos de actividad física y Cajas de Compensación, en los que se brinda, una modalidad de aeróbicos multinivel. Dicha modalidad, ha presentado casos que evidencian discordias y mal ambiente entre usuarios de diferentes niveles, del mismo nivel y hasta entre los usuarios y el docente que dirige la clase. Objetivo general: Relacionar las orientaciones de meta de los usuarios de la clase de aeróbicos multinivel, de una caja de compensación familiar, la afinidad por la didáctica empleada en clase y las características propias del docente. Metodología: La investigación presenta un enfoque empírico analítico, con un diseño no experimental, para el cual, se aplicaron dos instrumentos (TEOSQ y AUDCCDA), validados a través de juicio de expertos, a una población de 120 usuarios, practicantes de aeróbicos. Resultados: Las mujeres presentan una mayor participación que los hombres; los aeróbicos son una práctica sistemática en la que participan, en gran medida, adultos jóvenes; las orientaciones motivacionales no presentan una relación fuerte con los gustos o afinidades de los usuarios hacia un docente. Conclusiones: LoS usuarios muestran una clara orientación motivacional dirigida hacia la tarea; en cuanto a su afinidad son más destacadas en aquellos docentes carismáticos, novedosos y que muestren un estilo de enseñanza coherente y progresivo.

Palabras clave: Motivación; Afinidad docente; Didáctica; Ego; Tarea.

\section{ABSTRACT}

Introduction: Aerobics are a group and musical practice of physical exercise, whose objective is to work on the cardiovascular system, motor memory and coordination. In the city of Bogotá, this practice is very popular in places such as: gyms, physical activity institutes and compensation funds, in which a multi-level aerobic modality is offered. This modality has presented cases that show discord and bad atmosphere between users of different levels, of the same level and even between users 
and the teacher who directs the class. Generate objective: To relate $\mathrm{t}$ he goal orientations of the users of the multilevel aerobics $c$ lass o $f$ a family compensation $f$ und, $t$ he affinity for the didactics used in class and the characteristics of the teacher.

Methodology: The research presents an analytical empirical approach with a non-experimental design, for which two instruments (TEOSQ and AUDCCDA), validated through expert judgment, were applied to a population of 120 aerobics users. Results: Women present a greater participation th an me $n$, aerobics are a systematic practice in wh ich young adults participate to a great extent, motivational orientations do not present a strong relationship with the tastes or affinities of users towards a teacher. Conclusions: The users show a clear motivational orientation directed towards the task, in terms of their affinity, these are more prominent in those charismatic, novel teachers who show a coherent and progressive teaching style.

Keywords: Motivation; Teaching affinity; Didactics; Ego; Task.

\section{INTRODUCCIÓN}

Los aerobics o aeróbicos, en español, de acuerdo con algunos autores, se pueden definir como una actividad física coreografía al ritmo de la música, con diferentes modalidades, de mayor a menor intensidad: Alto- bajo impacto y de diferentes estilos, según Hermoso (1998). Los aeróbicos, también son considerados como una actividad gimnastica, generalmente, estructurada en secuencias lógicas o coreográficas, que se desarrollan al ritmo de la música, con el objetivo de conseguir una serie de beneficios en el plano psicofísico, propuesto por Diéguiz Papi (2004). Esta modalidad de ejercicio físico es muy popular en los últimos tiempos, dado sus componentes de estructuración, la música, las coreografías, los retos y la diversión que representan para algunos; por esto, muchas personas eligen realizar esta práctica y se mantienen en el tiempo; pero, hay muchas personas que desisten o no se atreven a involucrarse en ella, dada la exigencia en aspectos, como la coordinación, la concentración y la memoria motriz que implica. Es por esto, que este documento se interesó por caracterizar, de forma científica, las percepciones motivacionales de individuos, que practican esta modalidad de actividad física, en un lugar determinado.
La presente investigación tiene como propósito fundamental relacionar las orientaciones de meta de los usuarios de la clase de aeróbicos multinivel, de una caja de compensación familiar de Bogotá, la afinidad por la didáctica empleada en clase y las características propias del docente. Dicho proceso, se realizó a través de la implementación de dos cuestionarios; el primero: el cuestionario elaborado para determinar la orientación motivacional de un sujeto hacia una actividad determinada que, para el caso del estudio, se trata de los aeróbicos, como lo mencionan Duda \& Nicholls (1992), un cuestionario de orientación hacia la tare o el ego, TEOSQ, tomado de la versión de 16 ítems y traducida al español, por Sanchis Gimeno \& Peiró velert (2004), que presentó un proceso de ajuste y de revalidación, al contexto de la presente investigación: La actividad física grupal para la salud. El segundo instrumento, se denomina Cuestionario de Afinidad del Usuario a la Didáctica y Características del Docente de Aeróbicos, que fue creado y validado, para los propósitos de la presente investigación.

El desarrollo del presente ejercicio investigativo cuenta con un enfoque empírico analítico de investigación, con un diseño descriptivo correlacional. Durante el procedimiento de elaboración y construcción de la idea de investigación tuvieron lugar varios momentos; en primera medida, una revisión documental acerca del objeto de estudio, la evaluación y la caracterización de la motivación en prácticas relacionadas con el ejercicio y la actividad física para la salud, que presentó, entre otras situaciones, los instrumentos y las bases para la construcción del procedimiento a seguir; en un segundo momento, se llevó a cabo: 1) El ajuste, 2) La creación y 3) La validación de los instrumentos, bajo la modalidad de juicio de expertos. Luego de dicho proceso, se dio paso al tercer momento, que fue la aplicación de los instrumentos en la población participante de este proyecto y, por último, el análisis estadístico de los resultados, que permitió describir y establecer las correlaciones entre la información recolectada, logrando así, definir resultados y las conclusiones del estudio.

\section{MATERIALES Y MÉTODOS}

Esta investigación es de tipo descriptiva correlacional/ causal, en la cual, se describe la relación entre dos o más variables, en un momento determinado, 
mencionado por Hernández Sampieri et al. (2014). Presenta un enfoque empírico analítico, un diseño no experimental, es decir, aquella que se realiza sin manipular, deliberadamente, las variables y se observan fenómenos tal y como se dan en su contexto natural (Hernández Sampieri et al. 2014); para el caso de la presente investigación, se analizó la relación existente entre los datos sociodemográficos suministrados, los resultados obtenidos, a través del Cuestionario de la Orientación a la Tarea y al Ego (TEOSQ), constituido por 16 ítems, de los cuales, los 8 impares se encuentran orientados hacia el ego y los 8 pares, hacia la tarea y el cuestionador de afinidad del usuario a la didáctica y características del docente de aeróbicos, constituido por 29 ítems, divididos en cinco categorías (estilo de enseñanza, construcción coreográfica, ambiente en la clase, carisma del docente en clase e imagen del docente); los dos cuestionarios presentan una escala tipo Liker, de: 1 a 5, en la que uno (1) es totalmente en desacuerdo y cinco (5), totalmente de acuerdo.

Ambos instrumentos fueron sometidos a un respectivo proceso de valoración y de validación, mediante juicio de expertos, método utilizado para comprobar la fiabilidad de un instrumento en una investigación que, para este estudio, contó con los criterios de cinco Magísteres, con trayectoria y experiencia en el tema de investigación y actividad física grupal, quienes plantearon una serie de observaciones $y$ valoraciones, propias para el ajuste de los mismos (Escobar Pérez \& Cuervo Martínez, 2008).

Posterior al proceso de validación por expertos y a una prueba piloto con una muestra de 23 usuarios (3 hombres y 20 mujeres), los cuestionarios fueron enviados vía correo electrónico o vía WhatsApp, a las personas que participaron de las clases de aeróbicos, inscritos en los programas de clases grupales, de la caja de compensación, utilizando, como herramienta, la opción de formularios Google. En el mismo, se les indicó el plazo de 3 semanas para contestar, bajo su total autonomía y haciendo énfasis en la necesidad de contestarlo, con la mayor sinceridad posible.

Dicha información fue enviada a 120 individuos (98 mujeres y 22 hombres), usuarios practicantes de aeróbicos, de la caja de compensación, con edades comprendidas entre los 18 años en adelante, cuya experiencia en la práctica de los aeróbicos fuese de mínimo 2 meses y cuya asistencia a clases de aeróbicos, haya sido, por lo menos, una o dos veces por semana, durante el 2019.
Los análisis estadísticos de la presente investigación se realizaron a través del programa SPSS versión 25 estudiantil y programa Excel 2019. El análisis de la información inicial involucra medidas de estadística descriptiva, como porcentajes, promedios, frecuencias, mínimos, máximos y desviación estándar.

Finalmente, para el análisis de estadística inferencial, se utilizaron medidas como el coeficiente de Pearson, con el que se establecieron grados de correlación entre aspectos, como: 1) Los resultados sociodemográficos, 2) Los instrumentos de investigación y 3) Cada uno de los ítems desarrollados por cada instrumento.

\section{RESULTADOS Y DISCUSIÓN}

Consistencia interna de los instrumentos. El análisis de fiabilidad del cuestionario de afinidad del usuario a las didácticas y características del docente de aeróbicos, por un lado $y$, por el otro, del cuestionario TEOSQ modificado y restructurado para la clase de aeróbicos, se efectuó a través de la medida estadística, conocida con el nombre de Alpha de Cronbach. En primera instancia, se obtuvo el valor Alpha para el cuestionario TEOSQ, en la cual, el coeficiente de confiabilidad que se obtuvo fue de un (1) Alpha de 0,790; este valor generó la confiabilidad del instrumento para ser aplicado al presente estudio. Por otro lado, el Alpha para el cuestionario de afinidad del usuario a las didácticas y características del docente de aeróbicos, dio como resultado 0,633, lo que permitió generar una confiabilidad buena, para la implementación del cuestionario para estudio.

Análisis estadístico. Los análisis estadísticos de la investigación se realizaron a través del programa SPSS versión 25 estudiantil y programa Excel 2019. Para esta etapa de la investigación, se realizó un análisis de los resultados generados en este estudio. Se realizó un análisis descriptivo de las siguientes características de los usuarios participes de la investigación: edad, género, nivel de complejidad, al que asiste, durante las clases, número de días por semana, a los que asiste a clase, tiempo que lleva realizando esa práctica y se realizan otras actividades físicas.

De los 120 usuarios participantes, 98 fueron del género femenino y 22 del masculino, que representan un $81,7 \%$ y un 18,3\%, respectivamente; en cuanto a la edad de los usurarios, el mínimo fue de 18 años y el máximo 72 años, con un promedio de edad de 42,7 años $\pm 13,9$. 
Finalmente, la edad más destacada entre los usuarios es de 21 años, con una frecuencia de 6 y un porcentaje de 5 .

Para este estudio, los usuarios que participaron asistían a uno de los 3 niveles de complejidad, con los cuales, cuenta la clase de aeróbicos multinivel (básico, intermedio y avanzado). De los usuarios que practicaban aeróbicos multinivel, 14 pertenecian al nivel básico (1), 49 a nivel intermedio (2) y con una predominacia (moda) 57, al nivel avanzado, con un porcentaje de asistencia por nivel respectivo al: $11,7 \%, 40,8 \%$ y $47,5 \%$, respectivamente. En cuanto a la asistencia semanal durante el análisis de los datos, se encontró que, de las 120 personas, el mínimo de días, que asistían a clase era de uno (1) y el máximo de siete (7), con un promedio (media) de asistencia de $3,4 \pm 1,5$. Finalmente, la asistencia que más se repite con mayor frecuencia en los datos (moda) es 3 veces por semana. Respecto al tiempo de experiencia en la práctica de aeróbicos de los usuarios, el tiempo mínimo de práctica es de 0,48 años y el máximo, de 35 años. En cuanto al promedio (media) de experiencia es de 8,15 años $\pm 6,68$. Finalmente, los casos de experiencia que se repiten con mayor frecuencia en los datos (moda) son de 2,00 años.
Posteriormente, de acuerdo con los datos obtenidos de las prácticas con las que los usuarios complementan el trabajo físico, a parte de las clases de aeróbicos, teniendo en cuenta las frecuencias y los porcentajes de respuestas dadas por los usuarios, se evidencia que 81 usuarios practican otras clases grupales, como: complemento a su preparación física, que corresponde al 67,5\%; 30 realizan su complemento en el gym, correpondiente al 25,0\% y, finalmente, 9 usuarios realizaban otro tipo de prácticas deportivas, que corresponde al 7,5\%, de la población total.

En cuanto al análisis descriptivo realizado a los intrumento, para el caso del TEOSQ, los usuarios pertenecientes a cualquiera de los tres niveles de complejidad mostraron una clara y notoria orientación motivacional, dada hacia la tarea, es decir, de acuerdo con la relación en cuanto a la puntuación dada, los tres niveles presentaron una puntuación total de 5 , lo que indica, que están muy de acuerdo con cada uno de los ítems pares del instrumento TEOSQ. Interpretando el resultado, se puede destacar, cómo los usuarios realizan aeróbicos motivados por una satisfacción propia, ya sea por mejorar su condición física, sentirse mejor consigo mismo, entre otros (Tabla 1).

Tabla 1. Respuestas ítems orientados a la Tarea.

\begin{tabular}{|c|c|c|c|}
\hline \#Ítem & Moda Básico & Moda Intermedio & Moda Avanzado \\
\hline 2: & 5 & 5 & 5 \\
\hline 4: & 5 & 5 & 5 \\
\hline 6: & 5 & 5 & 5 \\
\hline 8: & 5 & 5 & 5 \\
\hline 10: & 5 & 5 & 5 \\
\hline 12: & 5 & 5 & 5 \\
\hline 14: & 5 & 5 & 5 \\
\hline 16: & 4 & 5 & 5 \\
\hline Moda total: & 5 & 5 & 5 \\
\hline
\end{tabular}

Aunque las puntuaciones dadas por los usuarios de los tres niveles fue la misma, para aquellos ítems orientados hacia la tarea (5 muy de acuerdo), también se destaca que, para aquellos ítems orientados hacia el ego, la puntuación en común que se evidenció fue de 3 (neutra), por lo cual, se podría decir, que existe alguna razón que dificulta a los usuarios, de cualquiera de los tres niveles, a dar una calificación concluyente (Tabla 2). 
Tabla 2. Respuestas ítems orientados al Ego.

\begin{tabular}{|c|c|c|c|}
\hline \#Ítem & Moda Básico & Moda Intermedio & Moda Avanzado \\
\hline 1: & 3 & 3 & 2 \\
\hline 3: & 3 & 4 & 3 \\
\hline $\mathbf{5 :}$ & 3 & 2 & 1 \\
\hline 7: & 2 & 1 & 1 \\
\hline 9: & 4 & 4 & 3 \\
\hline 11: & 3 & 3 & 3 \\
\hline 13: & 3 & 3 & 3 \\
\hline 15: & 3 & 3 & 3 \\
\hline Moda total: & 3 & 3 & 3 \\
\hline
\end{tabular}

Para el segundo cuestionario, afinidad del usuario por la didáctica y características del docente de aeróbicos, al interpretar los resultados de las puntuaciones dadas por los usuarios participes del estudio, en una escala Likert, de 1 a 5, se pudo encontrar que existe gran similitud en cada una de las puntuaciones, ya sea expresado por ítems, categorías o resultado total; el instrumento muestra un puntuación similar para los tres niveles, lo que permitiría deducir que, todos los usuarios, independiente de su nivel, buscan en el docente unas particularidades similares, en cuanto a su didáctica o características propias; un ejemplo de lo expuesto, se evidencia en la tabla 3, en donde se presentan las puntuaciones dadas por los usuarios, de los tres niveles, en cuanto a la primera categoría de dicho instrumento, denominada Estilo de enseñanza, conformada por 10 ítems, en los que se muestra una puntuación similar, entre los niveles de complejidad. Concluyendo que, para esta categoría, en general, los niveles básicos e intermedios, se encuentran totalmente de acuerdo con la categoría y avanzado, también se encuentra de acuerdo.

Tabla 3. Análisis de estilos de enseñanza y niveles de complejidad.

\begin{tabular}{|c|c|c|c|c|c|}
\hline 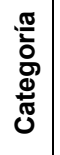 & \# ítem & Ítem &  & 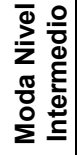 &  \\
\hline \multirow{10}{*}{ 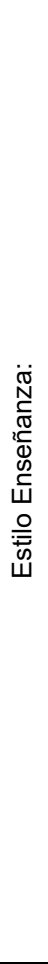 } & 1 & $\begin{array}{l}\text { La clase es más agradable cuando el profesor enseña una } \\
\text { coreografía paso a paso, es decir, del paso más sencillo } \\
\text { al paso más complejo }\end{array}$ & 5 & 5 & 4 \\
\hline & 2 & $\begin{array}{l}\text { La clase es más agradable cuando el profesor dedica } \\
\text { poco tiempo para enseñar la coreografía }\end{array}$ & 2 & 2 & 2 \\
\hline & 3 & $\begin{array}{l}\text { Me gusta cuando la clase es: fluida, coherente, segura, } \\
\text { resaltando la preparación de la clase por parte del } \\
\text { profesor }\end{array}$ & 5 & 5 & 5 \\
\hline & 4 & $\begin{array}{l}\text { Me gusta cuando la clase es: rápida, espontánea y } \\
\text { demostrando como pese a un olvido en determinado paso, } \\
\text { el profesor rápidamente tiene la capacidad de improvisar } \\
\text { uno nuevo }\end{array}$ & 4 & 4 & 4 \\
\hline & 5 & $\begin{array}{l}\text { Las coreografías son más fáciles de aprender si el } \\
\text { profesor utiliza señales verbales (indicaciones habladas } \\
\text { del paso o dirección) y visuales (indicaciones con las } \\
\text { manos) en cada paso }\end{array}$ & 4 & 5 & 5 \\
\hline & 6 & $\begin{array}{l}\text { El empoderamiento (la seguridad, confianza y dominio) } \\
\text { del docente contribuyen en el buen desarrollo de la clase }\end{array}$ & 5 & 5 & 5 \\
\hline & 7 & $\begin{array}{l}\text { Una postura encorvada, poco dialogo de instrucción y } \\
\text { movimientos pequeños por parte del profesor, pueden } \\
\text { afectar el ánimo y desarrollo de una clase }\end{array}$ & 5 & 5 & 5 \\
\hline & 8 & $\begin{array}{l}\text { Es agradable, cuando el profesor es equitativo con los } \\
\text { tiempos de enseñanza entre los grupos }\end{array}$ & 4 & 4 & 4 \\
\hline & 9 & $\begin{array}{l}\text { Es agradable, cuando el profesor tarda menor tiempo con } \\
\text { otros grupos y enfoca un poco más de trabajo con mi } \\
\text { grupo ya que puedo disfrutar más de la clase }\end{array}$ & 3 & 2 & 2 \\
\hline & 10 & $\begin{array}{l}\text { Me gusta, cuando mi profesor es paciente, se da cuenta } \\
\text { que aún no he aprendido el paso con facilidad, me da el } \\
\text { tiempo y repite la coreografía reforzar mi aprendizaje }\end{array}$ & 5 & 4 & 4 \\
\hline \multicolumn{3}{|c|}{ Moda total por categoría: } & 5 & 5 & 4 \\
\hline
\end{tabular}


En la tabla 4, se pueden evidenciar los puntajestotales, de acuerdo con cada una de las categorías del dados por cada uno de los niveles de complejidad, intrumento.

Tabla 4. Puntajes total dados por cada uno de los niveles de complejidad.

\begin{tabular}{|l|c|c|c|}
\hline \multicolumn{4}{|c|}{ Construcción Coreográfica } \\
\hline & Básico & Intermedio & Avanzado \\
\hline Moda: & $\mathbf{2}$ & $\mathbf{2}$ & $\mathbf{2}$ \\
\hline \multicolumn{5}{|c|}{ Ambiente en la Clase } \\
\hline & Básico & Intermedio & Avanzado \\
\hline Moda: & $\mathbf{3}$ & $\mathbf{3}$ & $\mathbf{3}$ \\
\hline \multicolumn{4}{|c|}{ Carisma del docente en clase y niveles de complejidad } \\
\hline \multicolumn{5}{|c|}{ Básico } & Intermedio & Avanzado \\
\hline Moda total: & $\mathbf{4}$ & $\mathbf{4}$ & $\mathbf{4}$ \\
\hline \multicolumn{4}{|c|}{ Imagen del docente en clase y niveles de complejidad. } \\
\hline \multicolumn{5}{|c|}{ Básico } & Intermedio & Avanzado \\
\hline Moda total: & $\mathbf{1}$ & $\mathbf{3}$ & $\mathbf{3}$ \\
\hline
\end{tabular}

Para esta investigación, la práctica de los aeróbicos es una de las tendencias en el fitness grupal y musicalizado, que sigue presentando gran fuerza y acogida en la participación del género femenino, a diferencia de la participación del género masculino, quienes, desde el nacimiento de estas prácticas, en los años 60, han venido mostrando comportamientos de participación similar, a los que se presentan en este estudio, tomando, como referencia, a Moral González et al. (2007).

En cuanto a la edad de participación de los usuarios en esta práctica, se pudo observar que los aeróbicos son una práctica que presenta gran afinidad y gusto en la población de adultos jóvenes, comprendida entre los 31 a 40 años de edad; son prácticas dirigidas a este tipo de población, sin escatimar la población joven, entre 21 a 30 años.

Por otra parte, cuando se relaciona la edad en promedio de las personas que realizan aeróbicos con la experiencia o tiempo que los usuarios llevan realizando esta práctica, la asistencia semanal de los usuarios a estas clases y las diferentes alternativas que los usuarios eligen al momento de realizar su práctica física, distintas a los aeróbicos, se puede evidenciar, cómo los aeróbicos son una práctica que, una vez sea aprendida y entendida, su disfrute es tal, que puede llegar a ser una actividad, cuya participación y gusto perdura en el tiempo, debido a que la tendencia en años de práctica de esta actividad, que se presenta en los resultados, así lo mostraron, al ver un promedio de participación temporal, enmarcado entre 1 a 7 años de experiencia, por un lado (con una frecuencia de 64 y un promedio de $53,3 \%$ ).

Al evidenciar los resultados obtenidos por el análisis generado en el apartado de prácticas con las que se complementa la clase de aeróbicos, se determina que las personas prefieren complementar su trabajo físico, con otras clases grupales, a parte de la clase de aeróbicos, en lugar de otros trabajos, como prácticas deportivas en gimnasio.

Por otra parte, teniendo en cuenta, el análisis inferencial realizado entre algunas características de la población, mediante el coeficiente de correlación de Pearson y el grado de significancia, se pudo determinar que las relaciones o asociaciones dadas entre determinadas características (nivel de complejidad, género, edad, experiencia en cuanto a la práctica de los aeróbicos) es débil, es decir, que el grado de asociación que presentan entre sí, las variables socio-demográficas son muy bajas. En otras palabras, el cambio de una de las variables no influirá de forma significativa en la otra, probablemente, se deba a la gran variedad de datos mostrados, por la población de estudio (Anexo 1). 
Anexo 1. Correlaciones entre algunos aspectos sociodemográficos

\begin{tabular}{|c|c|c|}
\hline \multicolumn{3}{|c|}{ Correlación: Genero VS Nivel de complejidad } \\
\hline & Género & Nivel de complejidad \\
\hline Correlación de Pearson & 1 & $0,275^{* *}$ \\
\hline Sig. (bilateral) & & 0,002 \\
\hline \multicolumn{3}{|c|}{ Correlaciones: Veces por Semana VS Nivel de Complejidad } \\
\hline & Nivel de complejidad & veces por semana \\
\hline Correlación de Pearson & 1 & 0,281 \\
\hline Sig. (bilateral) & & 0.002 \\
\hline \multicolumn{3}{|c|}{ Correlaciones: Nivel de Complejidad vs Tiempo de practica } \\
\hline & Nivel de complejidad & Tiempo de practica \\
\hline Correlación de Pearson & 1 & 0,005 \\
\hline Sig. (bilateral) & & 0,958 \\
\hline \multicolumn{3}{|c|}{ Correlaciones Nivel de Complejidad VS Edad } \\
\hline & Nivel de complejidad & Edad \\
\hline Correlación de Pearson & 1 & $-0,425^{* *}$ \\
\hline Sig. (bilateral) & & 0,000 \\
\hline
\end{tabular}

Al hacer un análisis más profundo de los resultados dados, tanto para las orientaciones por la tarea como por el ego, es claro que la mayor parte de usuarios afirmaron que la práctica de los aeróbicos, en general, la ejecutan motivados por un beneficio propio y cuyo interés está basado en la mejora y el aprendizaje de cada persona, para consigo mismo; pero al tomar los resultados en común, dado en la puntuación hacia el ego, como se dijo anteriormente, se evidencia que existe una puntuación muy marcada hacia la neutralidad, por lo que los usuarios no presentaron una afirmación concreta de lo que se piensa o no del ítem.

De otro lado, al revisar las puntuaciones obtenidas de cada una de las cinco categorías del segundo instrumento, Afinidad del usuario por la didáctica y características del docente de aeróbicos, se aprecia, en la primera categoría: estilo de enseñanza, se pretendía determinar cómo un usuario se identifica o qué características busca o quiere un usuario, en cuanto a estilos de enseñanza del docente, al momento de elegir una clase de aeróbicos, para esta categoría, que cuenta con 10 ítems; se presentó una puntuación en común de 5, para básico e intermedio y 4, para avanzado; se puede decir, entonces, que los usuarios de los tres niveles tienden a elegir las mismas características en un docente, a la hora de determinar su estilo de enseñanza. Es interesante observar cómo en el ítem 9 (Es agradable, cuando el profesor tarda menos tiempo con otros grupos y enfoca un poco más de trabajo con nuestro grupo, debido a que se puede disfrutar más de la clase), los niveles intermedio y avanzado dieron una puntuación de 2 (en desacuerdo), mientras que, en el nivel básico, se obtuvo una puntuación de 3 (neutro).

Para el ítem 2 (La clase es más agradable, cuando el profesor dedica poco tiempo para enseñar la coreografía), se observa que la opinión dada por los usuarios, de acuerdo con su nivel es similar (en desacuerdo), sería interesante complementar este análisis estadístico, dado por un cuestionario, con un trabajo de observación, debido a que, si se tiene en cuenta la información suministrada por diferentes docentes, que imparten esta clase, usualmente, los usuarios exigen menor tiempo para los grupos diferentes y mayor para ellos mismos, información que contradice, lo obtenido en el análisis.

Por otro lado, la puntuación en común dada para la categoría dos (construcción coreográfica), muestra una puntuación de 2 (en desacuerdo), que predomina en los tres grupos; en esta puntuación 
se destaca, la obtenida por los 11 ítems (Se prefiere cuando el profesor hace pasos que ya se conocen y no cambia su estilo, porque permite realizar la coreografía de una manera más rápida y fácil) y con la misma puntuación, se encuentra el ítem 16 (No gusta cuando el profesor implementa combinaciones o participaciones diferentes a las que ya se conocen); en teoría, la población de estudio, ante su respuesta, está comunicando que les agradan más las clases novedosas, únicas y diferentes, opiniones que también se recomendarían sea contrastada con algún tipo de análisis de campo y observación, para determinar, exactamente, si lo que se responde es directamente proporcional a lo que se hace.

Respecto a la categoría 3 (el ambiente de la clase), son pocos los ítems que conforman esta categoría, por ende, su evaluación y puntuación se ven un poco afectadas al momento de determinar algunas respuestas en común, para toda la categoría en especial; sin embargo, se puede afirmar que, para los usuarios de los tres niveles, la música o pistas musicales infiere, en gran medida, para un buen ambiente en clase, expresando su gusto por herramientas musicales novedosas, actuales y latinas. Al igual que lo ocurrido, en la anterior categoría, para la categoría 4 (el carisma del docente en clase), se puede afirmar que, de acuerdo con las puntuaciones dadas, por los niveles en cada uno de los ítems, al momento de ingresar a clase con un docente, ellos esperan que sea alegre, dinámico, con un tono de voz elevado, mas no descontrolado; básicamente, todos están de acuerdo en que la personalidad y el carisma del docente influye mucho en la decisión de ingresar a clase.
Finalmente, se encuentra la categoría 5 (imagen del docente), en la que se evidenció una puntuación en común neutra de los ítems. Para esta categoría, los usuarios de los tres niveles, no mostraron una opinión directa frente al contenido del ítem, por ejemplo, se encuentra el caso del ítem 24, en el que se le indica al usuario su afinidad por ingresar a clase con profesores del género masculino, a lo que básicamente se dio una respuesta de Muy en desacuerdo, misma puntuación dada para el ítem 23, que indica la preferencia por entrar a clase con profesoras mujeres; para el caso de intermedio y avanzado, dichos ítems obtuvieron una puntuación de 3.

Por otra parte, al detallar el análisis inferencial, en que se realizó una correlación de Pearson, entre las variantes de ego y tarea del instrumento TEOSQ vs las características sociodemográficas de los usuarios, no se evidenció ningún tipo de asociación o relación fuerte, ni moderada entre los ítems, que podría indicar que los aspectos sociodemográficos no son determinantes en la orientación motivacional de los usuarios, en la práctica de aeróbicos (Anexo 2). Situación similar, se presentó al momento de determinar la asociación o relación existente entre las variables sociodemográficas, con cada una de las categorías y sus respectivos ítems del cuestionario de afinidad hacia la didáctica y características del docente. Al realizar todo un análisis, solo se encontró un tipo de relación fuerte y directa entre el ítem 20, de la categoría 4 (carísima del docente) vs la variable edad, quienes obtuvieron una puntuación de 0,951 y que, en otras palabras, esto indicaría que la edad influye en la elección de un docente más comunicativo y expresivo. Básicamente, se podría decir, que la relación que se determinó entre la categoría y la variable fue directamente proporcional (Anexo 3).

\section{Anexo 2. Correlación de Pearson entre factores sociodemográficos vs TEOSQ}

\begin{tabular}{|c|c|c|c|c|c|c|c|c|c|}
\hline \multicolumn{10}{|c|}{ Correlación nivel de complejidad vs. ego } \\
\hline & $\begin{array}{c}\text { Nivel de } \\
\text { complejidad }\end{array}$ & $\begin{array}{c}\text { Item } \\
1 \\
\end{array}$ & $\begin{array}{c}\text { Item } \\
3 \\
\end{array}$ & $\begin{array}{l}\text { Item } \\
5\end{array}$ & $\begin{array}{c}\text { Item } \\
7\end{array}$ & $\begin{array}{c}\text { Item } \\
9 \\
\end{array}$ & $\begin{array}{c}\text { Item } \\
11\end{array}$ & $\begin{array}{c}\text { Item } \\
13 \\
\end{array}$ & $\begin{array}{c}\text { Item } \\
15\end{array}$ \\
\hline $\begin{array}{c}\text { Correlación de } \\
\text { Pearson }\end{array}$ & 1 & $-0,045$ & $-0,164$ & 0,054 & 0,087 & $-0,047$ & $-0,152$ & 0,019 & 0,015 \\
\hline \multicolumn{10}{|c|}{ Correlación nivel de complejidad vs. tarea } \\
\hline & $\begin{array}{c}\text { Nivel de } \\
\text { complejidad }\end{array}$ & $\begin{array}{l}\text { Item } \\
2 \\
\end{array}$ & $\begin{array}{c}\text { Item } \\
4\end{array}$ & $\begin{array}{l}\text { Item } \\
6 \\
\end{array}$ & $\begin{array}{c}\text { Item } \\
8\end{array}$ & $\begin{array}{c}\text { Item } \\
10 \\
\end{array}$ & $\begin{array}{c}\text { Item } \\
12 \\
\end{array}$ & $\begin{array}{c}\text { Item } \\
14\end{array}$ & $\begin{array}{c}\text { Item } \\
16 \\
\end{array}$ \\
\hline $\begin{array}{l}\text { Correlación de } \\
\text { Pearson }\end{array}$ & 1 & 0,035 & $-0,053$ & 0,032 & 0,109 & $-0,004$ & $-0,046$ & $-0,086$ & $-0,021$ \\
\hline \multicolumn{10}{|c|}{ Correlación nivel de practica vs. tarea } \\
\hline & $\begin{array}{c}\text { Nivel de } \\
\text { complejidad }\end{array}$ & $\begin{array}{l}\text { Item } \\
2 \\
\end{array}$ & $\begin{array}{c}\text { Item } \\
4\end{array}$ & $\begin{array}{l}\text { Item } \\
6 \\
\end{array}$ & $\begin{array}{c}\text { Item } \\
8 \\
\end{array}$ & $\begin{array}{c}\text { Item } \\
10 \\
\end{array}$ & $\begin{array}{c}\text { Item } \\
12 \\
\end{array}$ & $\begin{array}{c}\text { Item } \\
14\end{array}$ & $\begin{array}{c}\text { Item } \\
16 \\
\end{array}$ \\
\hline $\begin{array}{l}\text { Correlación de } \\
\text { Pearson }\end{array}$ & 1 & $-0,044$ & $-0,099$ & $-0,046$ & $-0,029$ & $-0,103$ & $-0,013$ & 0,078 & 0,039 \\
\hline
\end{tabular}




\section{Continuación anexo 2.}

\begin{tabular}{|c|c|c|c|c|c|c|c|c|c|}
\hline \multicolumn{10}{|c|}{ Correlación nivel de practica vs. ego } \\
\hline & $\begin{array}{c}\text { Nivel de } \\
\text { complejidad }\end{array}$ & $\begin{array}{c}\text { Item } \\
1\end{array}$ & $\begin{array}{c}\text { Item } \\
3\end{array}$ & $\begin{array}{l}\text { Item } \\
5\end{array}$ & $\begin{array}{c}\text { Item } \\
7\end{array}$ & $\begin{array}{c}\text { Item } \\
9\end{array}$ & $\begin{array}{c}\text { Item } \\
11\end{array}$ & $\begin{array}{c}\text { Item } \\
13\end{array}$ & $\begin{array}{c}\text { Item } \\
15\end{array}$ \\
\hline $\begin{array}{l}\text { Correlación de } \\
\text { Pearson }\end{array}$ & 1 & $-0,039$ & $-0,065$ & 0,046 & 0,067 & 0,028 & $-0,101$ & $-0,022$ & $-0,051$ \\
\hline \multicolumn{10}{|c|}{ Correlación nivel de edad vs. ego } \\
\hline & $\begin{array}{c}\text { Nivel de } \\
\text { complejidad }\end{array}$ & $\begin{array}{c}\text { Item } \\
1\end{array}$ & $\begin{array}{c}\text { Item } \\
3\end{array}$ & $\begin{array}{l}\text { Item } \\
5\end{array}$ & $\begin{array}{l}\text { Item } \\
7\end{array}$ & $\begin{array}{c}\text { Item } \\
9\end{array}$ & $\begin{array}{c}\text { Item } \\
11 \\
\end{array}$ & $\begin{array}{c}\text { Item } \\
13 \\
\end{array}$ & $\begin{array}{c}\text { Item } \\
15\end{array}$ \\
\hline $\begin{array}{l}\text { Correlación de } \\
\text { Pearson }\end{array}$ & 1 & 0,097 & 0,067 & 0,021 & $-0,091$ & 0,065 & 0,055 & 0,025 & $-0,019$ \\
\hline \multicolumn{10}{|c|}{ Correlación nivel de edad vs. tarea } \\
\hline & $\begin{array}{c}\text { Nivel de } \\
\text { complejidad }\end{array}$ & $\begin{array}{l}\text { Item } \\
2\end{array}$ & $\begin{array}{c}\text { Item } \\
4\end{array}$ & $\begin{array}{c}\text { Item } \\
6\end{array}$ & $\begin{array}{l}\text { Item } \\
8\end{array}$ & $\begin{array}{c}\text { Item } \\
10\end{array}$ & $\begin{array}{c}\text { Item } \\
12\end{array}$ & $\begin{array}{c}\text { Item } \\
14\end{array}$ & $\begin{array}{c}\text { Item } \\
16\end{array}$ \\
\hline $\begin{array}{l}\text { Correlación de } \\
\text { Pearson }\end{array}$ & 1 & 0,012 & 0,063 & 0,075 & 0,043 & 0,044 & 0,134 & 0,174 & 0,142 \\
\hline
\end{tabular}

Anexo 3. Correlaciones de Pearson entre aspectos sociodemográficos vs categoría 4: carisma del docente.

\begin{tabular}{|c|c|c|c|c|}
\hline \multicolumn{5}{|c|}{ Correlación Nivel de complejidad vs Carisma del profesor en clase } \\
\hline & $\begin{array}{c}\text { Nivel de } \\
\text { complejidad }\end{array}$ & ítem 19 & ítem 20 & ítem 21 \\
\hline $\begin{array}{l}\text { Correlación de } \\
\text { Pearson }\end{array}$ & 1 & 0,162 & $-0,387^{* *}$ & $-0,008$ \\
\hline \multicolumn{5}{|c|}{ Correlación Tiempo de practica vs Carisma del profesor en clase } \\
\hline & $\begin{array}{l}\text { Tiempo de } \\
\text { practica }\end{array}$ & ítem 19 & ítem 20 & ítem 21 \\
\hline $\begin{array}{l}\text { Correlación de } \\
\text { Pearson }\end{array}$ & 1 & $-0,170$ & $0,292^{* *}$ & 0,103 \\
\hline \multicolumn{5}{|c|}{ Correlación Edad vs Carisma del profesor en clase } \\
\hline & Edad & ítem 19 & ítem 20 & ítem 21 \\
\hline $\begin{array}{l}\text { Correlación de } \\
\text { Pearson }\end{array}$ & 1 & $-0,225^{*}$ & $0,951^{* *}$ & $-0,118$ \\
\hline
\end{tabular}

Finalmente, al realizar el análisis inferencial y determinar las posibles correlaciones entre los dos instrumentos del presente estudio: TEOSQ vs Afinidad por la Didáctica y Características del Docente, no se evidenció ningún tipo de relación de los resultados obtenidos. Al tomar ítem vs ítem o ítem vs Categoría, no se muestran asociaciones o correlaciones fuertes, ni moderadas, que permitan aplicar, de nuevo, ambos estudios, de manera conjunta. Al no determinar ninguna relación entre los instrumentos, se hace necesario, para futuros estudios, detallar que cada instrumento se debe tomar de manera independiente.

\section{CONCLUSIONES}

En consecuencia, con el análisis descriptivo sobre aquellos aspectos sociodemográficos de los usuarios que practican aeróbicos multinivel, de una caja de compensación en Bogotá, se determinó que, en su mayoría, son de género femenino; el nivel de complejidad, con mayor participación en el estudio, fue el nivel avanzado, seguido del nivel intermedio y finalmente, el básico, con una participación baja. Se identifica que los aeróbicos son una práctica que se ejecuta, por la mayoría de usuarios, como mínimo 3 veces a la semana, lo que la convierte en una práctica sistemática y de alto valor, a la hora de complementar un trabajo físico o un tipo de entrenamiento.

También, se pudo determinar que, para la mayoría de personas que practican aeróbicos, en esta caja de compensación, ven en las clases grupales un medio para llevar a cabo su práctica o entrenamiento físico, lo que evidencia el gusto de las personas por hacer 
actividades de forma social y conjunta, seguidos por un docente y, en su mayoría, al ritmo de la música, situación que al profundizarse, sería de gran interés poder ahondar acerca de las alternativas o comportamientos, que este tipo de población, afín a las clases grupales, ha presentado, en tiempos de pandemia, en que la virtualidad, el distanciamiento y la baja interacción social, son protagonistas en esta nueva normalidad. En términos de experiencia, se evidencia que los aeróbicos son una actividad que se aprende desde joven y que, con la práctica y el gusto, llegan a ser una acción que perdura en el tiempo.

En cuanto al análisis realizado a los instrumentos de investigación, se determinó, respecto al instrumento de orientación motivacional TEOSQ, que los usuarios de esta caja de compensación realizan aeróbicos basados en una motivación propia, es decir, el objetivo de practicar esta clase es mejorar su condición física, sentirse bien consigo mismo, aprender nuevos movimientos, entre otros; en definitiva, de acuerdo con lo expresado por ellos mismos, en sus respuestas, se muestra una alta afinidad por una orientación motivacional, dada hacia la tarea.

Pese a la puntuación y la orientación motivacional expresada por los usuarios al responder el cuestionario, es importante destacar la alta relación que se presentó en la puntuación, neutra, brindada por los usuarios, pertenecientes a los tres niveles de complejidad; con relación a los ítems orientados hacia el ego, en que se infiere que, pese a la alta orientación motivacional mostrada por la población, dirigida hacia la tarea, es importante realizar un proceso de reflexión del trabajo docente a la hora de preparar e impartir una clase de aeróbicos, reflexión en que se puedan generar herramientas y alternativas, que promuevan y fortalezcan un mayor trabajo, que busque la orientación hacia un objetivo más intrínseco en el usuario y, de esta manera, se pueda mejorar el clima motivacional en clase, la adhesión a la misma, el respeto por el compañero y el docente $y$, finalmente, el gusto por la ejecución de este tipo de prácticas.

Del instrumento de Afinidad por la Didáctica y las Características del Docente, se indica que, la mayoría de respuestas dadas por los usuarios en los ítems, fueron similares, en cuanto a su puntuación, destacando la gran acogida y valor que, para los usuarios, presentan aquellos docentes que siempre buscan realizar cosas novedosas, preparan su clase con antelación, son animados, activos y extrovertidos, buscan siempre estar a la vanguardia en métodos de construcción coreográfica y diseño de pasos, respetan los tiempos de enseñanza coreográfica entre los grupos, evidenciando así, una equidad por la clase. Se destaca la no importancia por el género del docente, es decir, que las personas ingresan a clase sin importar, si el docente es hombre o mujer, situación similar ocurre al momento de ingresar a una clase, cuyo docente sea atlético o no.

Básicamente, las respuestas dadas por los usuarios tienden a ser totalmente fraternales, cordiales, amables, desinteresadas y comprensibles para el docente; pero, en ese orden de ideas, si, según lo plasmado por el usuario, todo es como debería ser, no se encuentra una relación entre las respuestas obtenidas y la información suministrada por la experiencia docente y los mismos participantes. En este punto, también se destaca, en algunos ítems, una puntuación que no permite evidenciar, si el usuario está de acuerdo o no con el ítem y así poder entrar a hacer un análisis más elaborado; lo anterior ocurre en situaciones, como, por ejemplo, el ítem 28 (Prefiero ingresar a las clases de los profesores con menor antigüedad), que tiene un predominio de calificación neutra, al relacionar este tipo de puntajes, con otros ítems que presentan la misma situación; se podría concluir, que no es totalmente claro el discurso del usuario y podría parecer que limita sus respuestas, de acuerdo con una conciencia enmarcada por un juicio socialmente establecido.

Por otro lado, de acuerdo con los resultados obtenidos del análisis correlacional, se evidencia una correlación débil entre las orientaciones motivacionales dadas hacia el ego o la tarea y la afinidad que presenta el usuario con las didácticas y características propias del docente de aeróbicos, en que se presenta una correlación débil, es decir, que la correlación dada entre ambos aspectos a investigar, no permite realizar un análisis más detallado y poder dar mayor claridad a interrogantes planteados. Teniendo en cuenta esta idea y los análisis destacados anteriormente, se concluye que, para determinar una respuesta con mayor veracidad y detalle, en cuanto a las orientaciones motivacionales que llevan a un usuario a realizar una práctica, por un lado y, por el otro, evidenciar qué apreciación, características y didácticas busca un usuario en un docente al momento de ingresar a su clase de aeróbicos, ambos estudios, para futuras investigaciones, se deberán realizar de forma separada. 
En correspondencia con lo anterior, la modificación y la validación del instrumento TEOSQ, ha mostrado ser una herramienta muy útil para investigaciones posteriores, que se pretendan realizar en el campo de las orientaciones motivacionales en la actividad física grupal y musicalizada que, al momento de emplearse en adultos, se recomienda complementar la investigación con otro tipo de herramientas presenciales, es decir, se aconseja realizar una comparación entre el discurso que brinda un usuario o persona de una temática determinada, con un análisis de campo y observación que el investigador realice de la población y, así evidenciar, si en realidad las respuestas dadas son coherentes a las dinámicas y comportamientos presentados por el individuo.

Finalmente, para el instrumento de Afinidad por la Didáctica y Características del Docente de Aeróbicos, es un cuestionario que permite ser el pilar o la base de estudios futuros, que pretendan evidenciar la realidad de lo que en verdad busca un individuo al ingresar y participar de una clase, impartida por determinado profesor; esto aplicaría no solo para una clase de aeróbicos, sino también, para cualquier tipo de clase grupal y musicalizada.

Como recomendaciones, por un lado y de acuerdo con su nivel de confiabilidad, dado en el análisis Alpha de Cronbach: 0.633, el instrumento muestra la necesidad de presentar diversas modificaciones, en cuanto a su estructura, organización de los ítems, número de categorías, igual número de ítems, de acuerdo con las categorías, mayor claridad en la construcción de los ítems, entre otros aspectos, con el fin de volver el instrumento más confiable y consistente, al momento de realizar una investigación. Por otro lado, y al igual con el caso del instrumento de TEOSQ, se recomienda, al realizar un trabajo de investigación, utilizando el instrumento de Afinidad, contrastarlo con otra herramienta, que permita evidenciar, de manera presencial, las acciones y los comportamientos de la población a estudiar, de tal manera, que se pueda contar con unos resultados más verídicos y concluyentes.

\section{Conflicto de intereses: El manuscrito fue preparado} y revisado con la participación de todos los autores, quienes declaramos que no existe ningún conflicto de intereses que ponga en riesgo la validez de los resultados presentados. El proyecto de investigación fue financiado por el autor.

\section{REFERENCIAS}

1. DIÉGUIZ PAPI, J. 2004. Aerobic en salas de fitness. Manual teórico práctico. (Segunda edición) Editorial INDE Publicaciones (Barcelona, España).168p.

2. DUDA, N.; NICHOLLS, J. 1992. Dimensions of Achievement Motivation in Schoolwork and Sport. Journal of Educational Psychology. 84(3):290-299.

3. esCOBAR PÉREZ, J.; CUERVO MARTínEZ, A. 2008. Validez de contenido y juicio de expertos: una aproximación a su utilización. Avances en Medición. 6:27-36.

4. HERMOSO VEGA, Y.M. 1998. El aerobic como mejora de la calidad de vida. Puertas a la lectura. 4:66-69.

5. HERNÁNDEZ SAMPIERI, R.; FERNÁNDEZ COLLADO, C.; BAPTISTA LUCIO, M. DEL P. 2014. Metodología de la investigación. sexta edición. Mc Graw Hill Education. México. 600p.

6. MORAL GONZÁLEZ, S.; FERNÁNDEZ GONZÁLEZ, I.; LÓPEZ ERQUICIA, B. 2007. Manual de aeróbic y step. Paidotribo. 168p.

7. SANCHIS GIMENO, J.R.; PEIRÓ VELERT, C. 2004. Propiedades psicométricas de la versión inicial del cuestionario de orientación a la tardea y al ego (TEOSQ) adaptado a la educación física en su traducción al castellano. Revista de Psicología del Deporte. 13(1):25-39. 